\title{
Apredendendo Física moderna por meio de um experimento de baixo custo: um relato de aula prática
}

\author{
Paulo José Pereira de Oliveira \\ Instituto Federal do Espírito Santo - Campus Cachoeiro (IFES) \\ (paulojoseo@ifes.edu.br) \\ Edmundo Rodrigues Júnior \\ Instituto Federal do Espírito Santo - Campus Cachoeiro (IFES) \\ (edmundor@ifes.edu.br) \\ Bruno Fernandes de Souza \\ Instituto Federal do Espírito Santo - Campus Cachoeiro (IFES) \\ (bfernadessousa@gmail.com) \\ Flávio Correa Prêmoli \\ Instituto Federal do Espírito Santo - Campus Cachoeiro (IFES) \\ (flaviocpremoli@gmail.com) \\ Gustavo Figueira Martini \\ Instituto Federal do Espírito Santo - Campus Cachoeiro (IFES) \\ (gu_martini@hotmail.com) \\ Rafael Figueira Martini \\ Instituto Federal do Espírito Santo - Campus Cachoeiro (IFES) \\ (rafaelfmartini@hotmail.com)
}

\begin{abstract}
Resumo: Neste trabalho apresento, utilizando materiais de baixo custo, o experimento de Young e o de difração por uma fenda circular, realizados por estudantes de engenharia com o objetivo de determinar o comprimento de onda de um laser caseiro. Para o experimento de Young, os valores obtidos do comprimento de onda foram 654,7 nm (máximo de primeira ordem, $\mathrm{m}=1$ ) e $645,84 \mathrm{~nm}$ (máximo de quinta ordem, $m=5$ ) um desvio de respectivamente $0,71 \%$ e $0,65 \%$ em relação ao valor de referência de $650 \mathrm{~nm}$, no rótulo do laser caseiro. O valor obtido no experimento de difração por uma fenda circular foi de $639,56 \mathrm{~nm}$, um desvio de apenas 1,6\%. Os resultados indicaram que é possível, utilizando qualquer um dos experimentos e materiais de baixo custo, obter com boa precisão, o comprimento de onda da luz. Igualmente esta atividade promoveu uma interação maior entre os estudantes e favoreceu o entendimento do caráter ondulatório da luz.
\end{abstract}

Palavras-chave: difração; interferência; comprimento de onda.

\section{Learning modern Physics by means of a low cost experiment: a practical class report}

Abstract: Using low cost materials, we present in this work the experiments of the Young's diffraction and of the diffraction by a circular aperture, made by engineering students, in order to determine the wavelength of a laser used at home. For Young's experiment, the values of the wavelength were 654.7 $\mathrm{nm}$ (maximum of the first order, $\mathrm{m}=1$ ) and $645.84 \mathrm{~nm}$ (maximum of fifth order, $\mathrm{m}=5$ ), a deviation of respectively $0.71 \%$ and $0.65 \%$ when compared with the reference value of $650 \mathrm{~nm}$ from the laser label. The value obtained at the diffraction by a circular aperture was of $639.56 \mathrm{~nm}$, a deviation of only $1.6 \%$. The results indicated that it is possible, using any of the experiments and low cost materials, to obtain with good accuracy, the wavelength of light. Furthermore, this activity promoted greater interaction between students and contributed to the understanding of the ondulatory nature of light.

Keywords: diffraction; interference; wavelength. 


\section{Introdução}

O uso de atividades experimentais como estratégia didática para ensinar conteúdos de Ciências tem despertado o interesse de muitos pesquisadores. A utilização dos experimentos na sala de aula pode proporcionar aos discentes "um contato real com os conteúdos ensinados, tornando-os, assim, mais interessantes" (MARINHO et. al., 2015, p.1).

Para Silva e Assis (2012) os estudantes podem ser incentivados pelo professor a investigarem o que acontece durante a execução de um experimento. Essa investigação pode acontecer, por meio de questionamentos direcionados aos estudantes para que eles expressem suas concepções alternativas na tentativa de explicarem o funcionamento do experimento proposto. "Cria-se, assim, um ambiente descontraído em sala de aula e estabelece-se uma interação social mais rica entre os alunos e o professor, o que, segundo Vigotsky (2003), favorece a aprendizagem" (SILVA e ASSIS, 2012, p.316).

Dentre os diversos tópicos do componente curricular Física que podem ser explicados com o auxilio das experiências, o entendimento da natureza ondulatória da luz permite a compreensão do espectro eletromagnético como ondas de rádio, Microondas, infravermelho, região do visível, dentre outros; tão presentes no nosso cotidiano por meio das aplicações tecnológicas. A compreensão da natureza ondulatória da luz permite o entendimento da relação entre o eletromagnetismo e a óptica (SOUZA et. al., 2015), obtida por meio das equações de Maxwell no século XIX.

Neste trabalho apresentamos o relato de uma aula prática para a determinação do comprimento de onda de um laser caseiro utilizando o experimento de Young e o da difração por fenda circular; ambos construídos com materiais de baixo custo. A intervenção didática foi realizada durante as aulas de Introdução a Física Moderna, com estudantes do $4^{\circ}$ período de Engenharia de Minas de uma instituição federal do Espírito Santo.

\section{Fundamentação teórica}

A formação do arco-íris, a mudança de cor nas asas de uma borboleta e em um CD, as cores em uma bolha de sabão e em uma mancha de óleo em uma pista 
são fenômenos provocados pela interferência de ondas luminosas: "A interferência é um dos mais típicos fenômenos ondulatórios, consistindo na superposição de ondas em um ponto do espaço" (RIBEIRO e VERDEAUX, 2012. p.7). Por meio do estudo do fenômeno da interferência é possível determinar, entre muitas coisas, o comprimento de onda da luz e o índice de refração de um dado material.

Em 1678, o cientista holandês Christian Huygens apresentou uma teoria de que a luz apresentava um caráter ondulatório, contrariando Isaac Newton que acreditava que a luz era formada por partículas. Utilizando construção geométrica, Huygens explicou as leis de reflexão e refração e atribuiu um significado físico ao índice de refração. Embora sua teoria seja muito menos completa do que as de James Clerk Maxwell, com a teoria eletromagnética, a teoria de Huygens era matematicamente mais simples e permanece útil até hoje.

Em 1801, Thomas Young demonstrou que a luz sofre interferência como as ondas do mar, as ondas sonoras e todos os outros tipos de ondas ${ }^{1}$. Embora extremamente importante para os estudos dos fenômenos ondulatórios, segundo Silva (2009), a experiência de Young não pode ser considerada definitiva em relação à validação da teoria ondulatória e à queda da corpuscular; mesmo porque na época de Young ainda não existia uma teoria matemática sólida para explicar o caráter ondulatório da onda. Isso viria a acontecer em 1864 com a unificação das teorias elétricas e magnéticas feitas por James Clerk Maxwell.

O fenômeno da interferência luminosa é o resultado da difração da luz.

"A difração ocorre quando a luz atravessa fendas ou se desvia de obstáculos de mesma ordem de grandeza do seu comprimento de onda (RIBEIRO e VERDEAUX, 2012, p.6)". Segundo Silva (2009) o crédito da descoberta do fenômeno da difração se deve ao físico jesuíta italiano Francesco Maria Grimaldi (1618-1663), que relatou esse fenômeno pela primeira vez na sua obra Physico-Mathesis de Lumine, Coloribus et Iride, publicado em 1665.

A compreensão do fenômeno da difração não é simples. Barbosa et. al. (2012) apresentam uma lista de referências que abordam o tema em diferentes níveis de complexidade que vai desde a abordagem mais simples; encontrada em um livro de graduação (vide: Halliday; Resnick e Walker, 2009) a um livro de teoria

\footnotetext{
1 Sem reportar aos gregos, autores como Hooke, Descartes e Euler, foram também importantes para o desenvolvimento da teoria ondulatória (SILVA, 2009).
} 
eletromagnética usado em cursos mais avançados (vide: Born e Wolf, 1999).

A fundamentação teórica sobre difração utilizada neste trabalho se baseou no nível do livro de graduação, suficiente para explicar os resultados encontrados nesta aula prática. Além disso, uma explicação menos complexa é mais acessível para os professores de física do ensino médio e até mesmo para docentes da graduação que não possuem especialidade em óptica.

\section{Entendendo o experimento de Young}

A figura 1 abaixo mostra um esboço do experimento realizado por Young:

Figura 1: Experimento de Young:

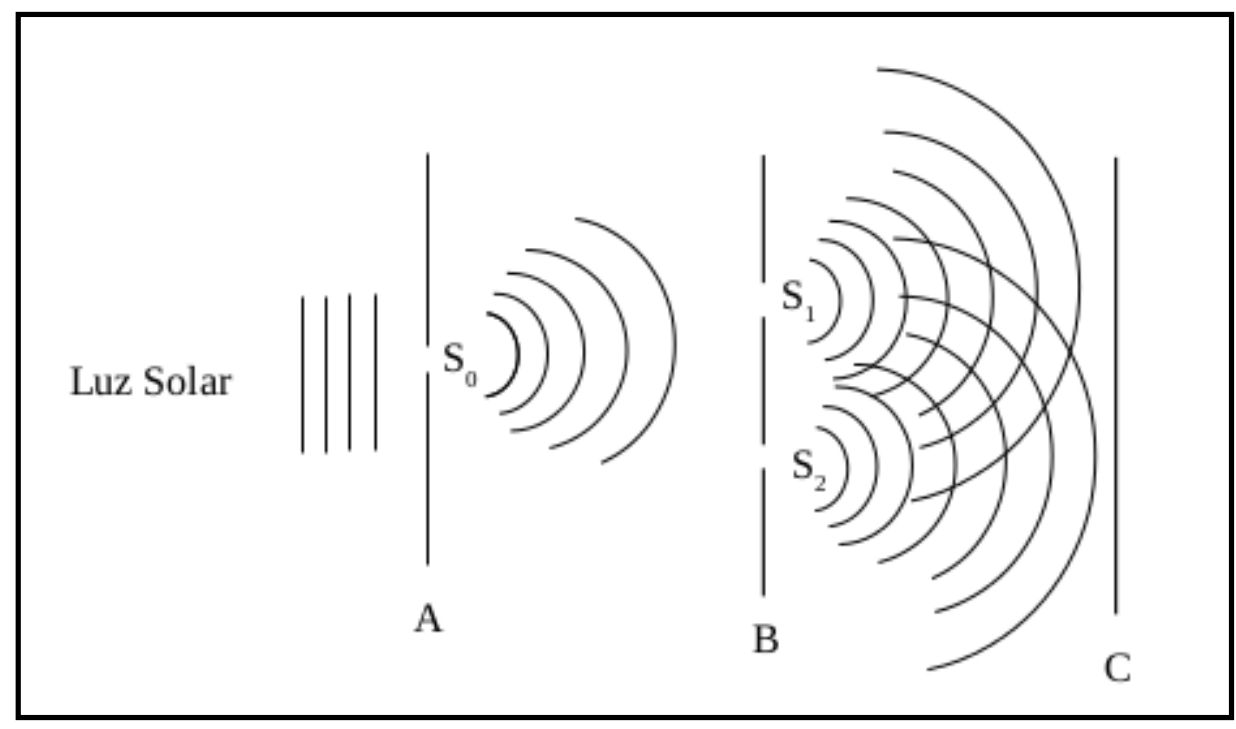

Fonte: adaptado de Halliday; Resnick e Walker, 2009

Uma fonte de Luz ilumina a fenda So do anteparo $A^{2}$. A luz difratada pela fenda se espalha e é usada para iluminar as fendas $S_{1}$ e $S_{2}$ do anteparo B. Uma nova difração ocorre quando a luz atravessa as duas fendas, e duas ondas se propagam simultaneamente no espaço à direita do anteparo $B$, interferindo uma com a outra. $A$ tela $\mathrm{C}$, é utilizada para observação da figura de interferência. A figura 2 mostra um

2 A presença do primeiro orifício na tela inicial deixa o feixe de luz mais coerente, e permite consequentemente, que o feixe luminoso proveniente do orifício So encontre com os demais orifícios $S_{1}$ e $S_{2}$ da segunda tela. Uma fonte de luz é coerente quando a diferença de fase proveniente da onda eletromagnética emitida por essa fonte permanece invariável por todo espaço (HALLIDAY; RESNICK e WALKER, 2009, p.86) 
esboço de uma figura de interferência produzida por um arranjo como o da figura 1:

Figura 2: Esboço de uma figura de interferência produzida em um anteparo de observação.

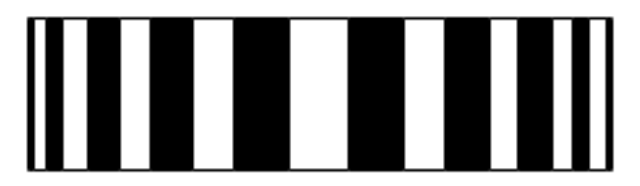

Fonte: adaptado de Halliday; Resnick e Walker, 2009.

Nesta figura, as faixas claras (brancas) correspondem às interferências construtivas e as faixas escuras as interferências destrutivas. No centro, tem-se uma faixa clara (chamada de máximo central) e a esquerda e direita do máximo central as faixas claras e escuras se alternam. A figura 3 mostra como Young encontrou uma expressão matemática para determinar a localização dessas faixas, também chamadas de pontos claros e escuros.

Figura 3: Raios luminosos partem das fendas $S_{1}$ e $S_{2}$ e se interferem em $P$.

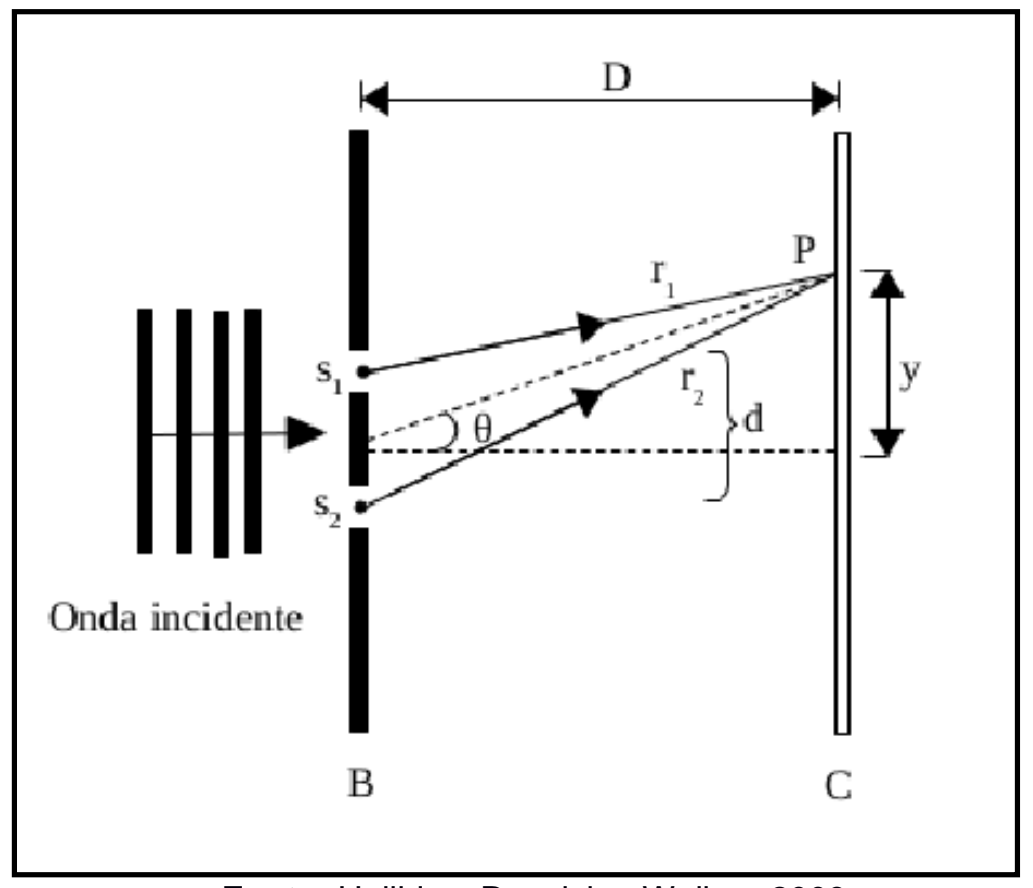

Fonte: Halliday; Resnick e Walker, 2009.

Nesta figura, $r_{1}$ e $r_{2}$, representam as ondas partindo das fontes $S_{1}$ e $S_{2}$ e $P$ representa a localização, em termos da altura y, dos pontos. Considerando que $D>>d$, podemos considerar os raios $r_{1}$ e r2 aproximadamente paralelos (figura 4). 
Figura 4: Determinação da localização dos pontos de interferência. Nesta figura, $\Delta \mathrm{L}$ é a diferença das distâncias percorridas pelos raios $r_{1}$ e $r_{2}$.

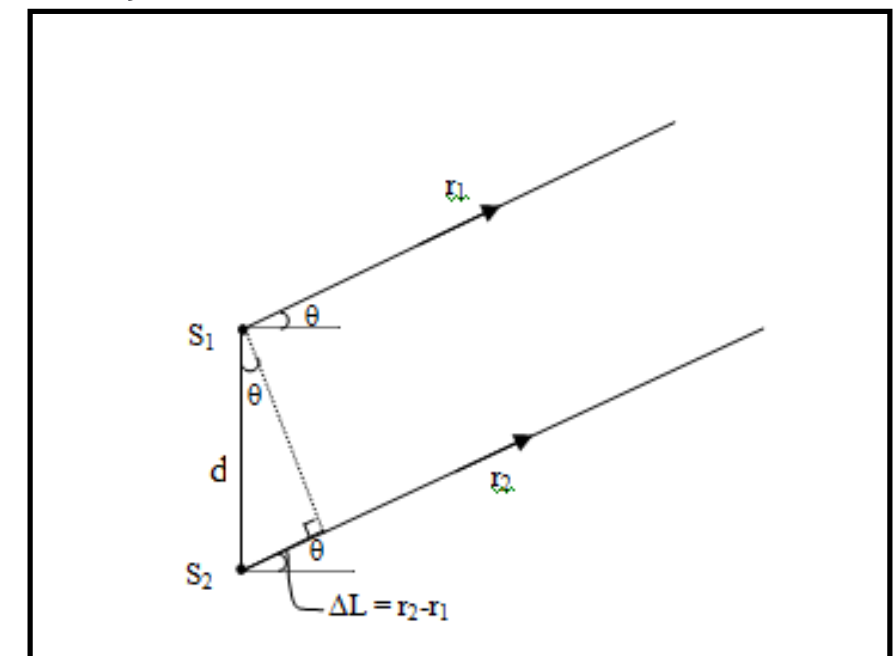

Fonte: Halliday; Resnick e Walker, 2009.

Conforme a figura 4, temos as seguintes expressões matemáticas para a localização das faixas claras e escuras:

$$
\begin{array}{r}
d \operatorname{sen} \theta=m \lambda \quad(\text { pontos claros) (1) e } \\
d \operatorname{sen} \theta=(m+1 / 2) \lambda \text { (pontos escuros) }
\end{array}
$$

onde $m=0,1,2,3 \ldots$, define a ordem $(m=1$, por exemplo, é chamado de ponto claro de primeira ordem e assim por diante).

Utilizando considerações geométricas, para um m qualquer, é possível associar a distância $\mathrm{D}$, a altura y, com o comprimento de onda. Sabendo que:

$$
\operatorname{tang} \theta=y / D
$$

concluímos que $\theta=\operatorname{tang}^{-1}(y / D)$. Substituindo $\theta$ em uma das equações (1) ou (2) podemos encontrar o comprimento de onda, para um $\mathrm{m}$ qualquer.

\section{Entendendo o experimento da difração por fenda circular}

Se no lugar de uma fenda, utilizarmos um orifício circular, a figura de interferência formada pela passagem da luz por este orifício terá uma forma semelhante à mostrada abaixo: 
Figura 5: Imagem de interferência gerada por um orifício circular.

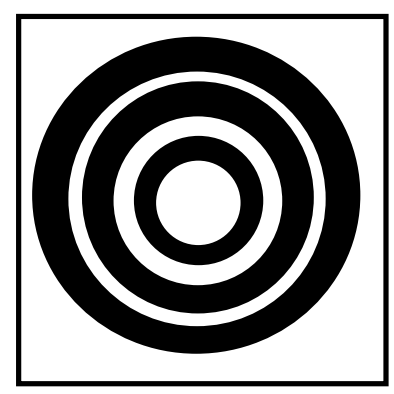

Fonte: Halliday; Resnick e Walker, 2009.

Com um círculo claro no centro (máximo central) e, mínimos e máximos circulares alternados. Para este caso a posição do primeiro mínimo (primeiro circulo escuro após o máximo central) é dada por:

$$
\operatorname{Sen} \theta=1,22 \lambda / d \text {. (4) }
$$

Onde $\theta$ é o ângulo entre o eixo central e a reta que liga o centro do anel à posição do mínimo circular. O diâmetro do orifício circular é dado por d e $\lambda$ é o comprimento de onda da luz incidente.

A seguir apresentamos a trajetória metodológica do nosso trabalho.

\section{Percurso metodológico}

As atividades práticas foram realizadas no primeiro semestre de 2014 com vinte estudantes distribuídos em quatro grupos de cinco. Cada grupo deveria apresentar um relatório sobre a aula experimental. Para a confecção deste trabalho foi selecionado apenas o relatório do grupo 1. Os critérios de seleção para tal escolha foram: a qualidade das figuras de difração e interferência obtidas nos experimentos; a qualidade das medidas e; clareza e aprofundamento da introdução, desenvolvimento e conclusão.

Para a realização do Experimento de Young e o da difração por uma fenda circular foram utilizados os seguintes materiais: pente de cabelo, fita isolante, mesa, anteparo, laser de luz vermelha, trena e régua e uma tampa de caneta (figuras 6 e 7) O experimento foi realizado em uma sala de aula e uma das paredes foi utilizada como anteparo. 
Figura 6: materiais utilizados no experimento de Young.

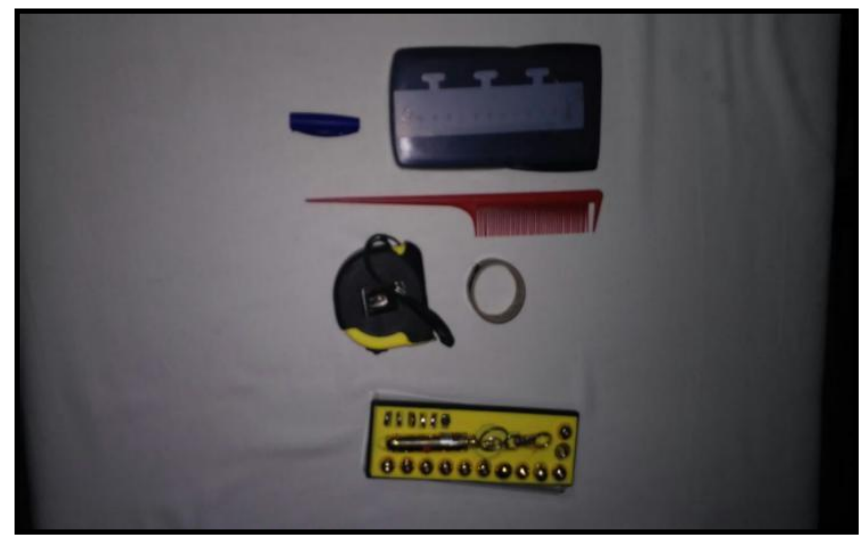

Fonte: arquivo pessoal, 2014.

Figura 7: tampa de caneta utilizada no experimento de difração.

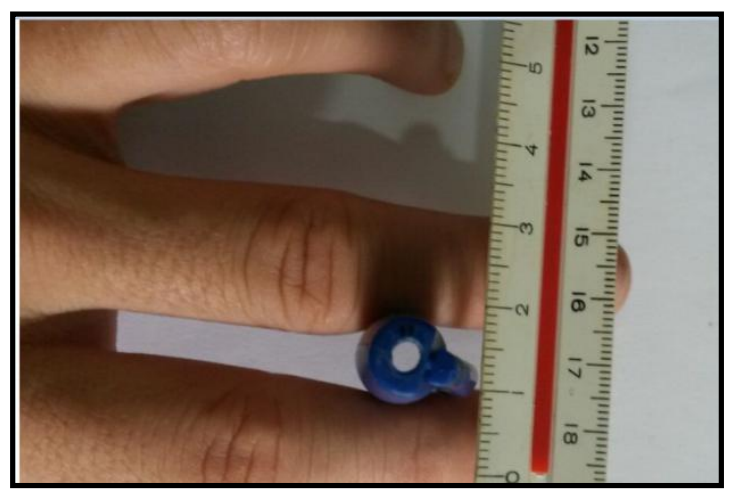

Fonte: arquivo pessoal, 2014.

\section{Experimento de Young}

Cobrimos os dentes do pente com uma tira de fita isolante para formar duas fendas isoladas e separadas por um dente. Com o auxilio de uma régua medimos a distância entre essas fendas e encontramos o valor de $(d=2 \mathrm{~mm})$. Utilizando outro pedaço de fita isolante fixamos o pente na vertical apoiado a uma mesa. Equilibramos o laser sobre a mesa e o direcionamos ao ponto médio entre as fendas do pente (como o laser emite uma luz coerente não houve a necessidade da primeira fenda inserida originalmente por Young em seu experimento). Medimos a distância (D) entre as fendas do pente e a parede (anteparo). A figura de interferência foi gerada, e com auxilio da régua, medimos a distância $(\mathrm{y})$ do ponto central da figura até o $1^{\circ}$ máximo $(\mathrm{m}=1)$ e também para o $5^{\circ}$ máximo $(\mathrm{m}=5)^{3}$ (ver figura 8$)$.

3 Os valores $m=1$ e $m=5$ foram escolhidos de forma aleatória pelos estudantes. 
Figura 8: franjas de interferência obtidas com o experimento de Young utilizando um laser caseiro.

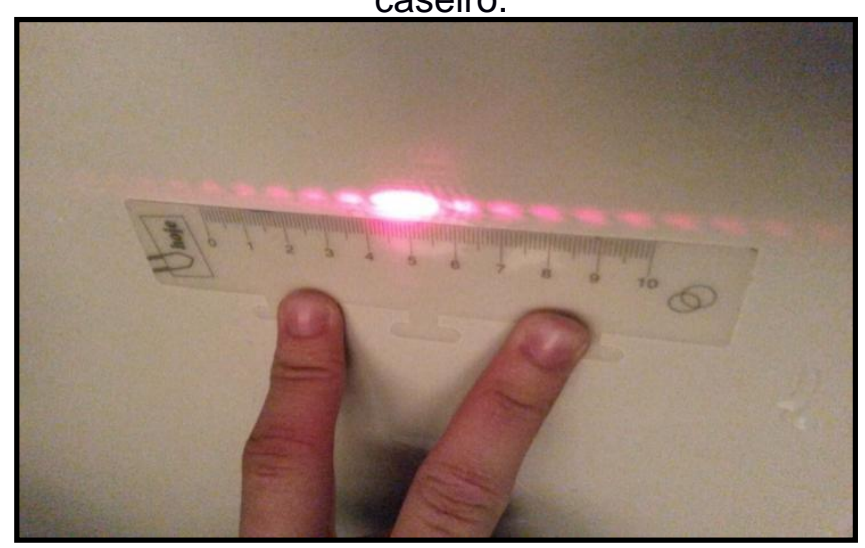

. Fonte: arquivo pessoal, 2014.

Após encontrar as distâncias, calculamos os valores de $\theta$ por meio da equação (3): Com os valores de $\theta$ e utilizando a equação (1) encontramos os valores de $\lambda$ para (m =1) e $(\mathrm{m}=5)($ ver tabela 1$)$.

\section{Experimento de difração por uma fenda circular}

Conforme mostramos na figura 7, fizemos um furo com diâmetro de $\mathrm{d}=2 \mathrm{~mm}$, em uma tampa de caneta circular. Com o auxílio de uma fita isolante, fixamos a tampa à mesa. Equilibramos o laser sobre a mesa, fazendo-o incidir no centro do furo da tampa da caneta. Medimos a distância (D) entre a tampa da caneta e a parede ou anteparo.

A figura de interferência foi gerada, e com auxilio da régua, medimos a distância do ponto central da figura até o $1^{\circ}$ mínimo $(m=1)$. Abaixo segue uma foto da figura gerada:

Figura 9: franjas de interferência gerada por uma fenda circular com o auxilio de um laser caseiro.

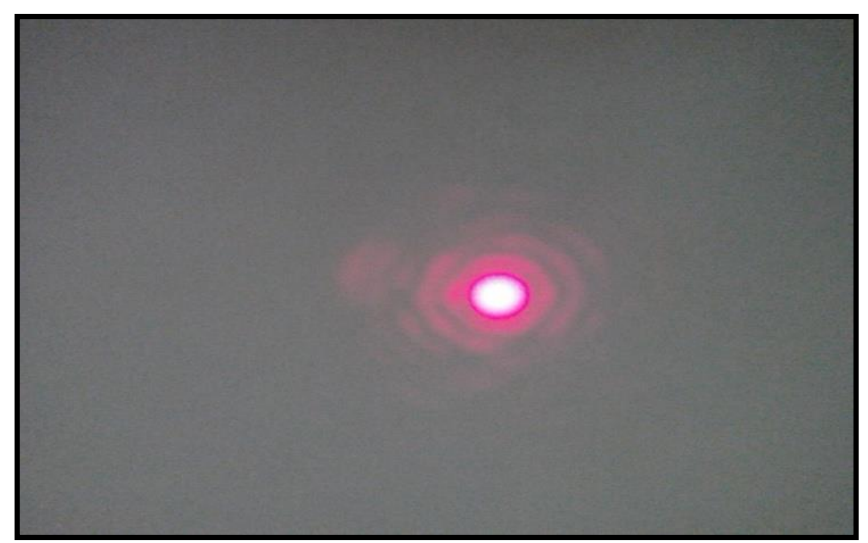

Fonte: arquivo pessoal, 2014. 
Após encontrar as distâncias, calculamos os valores de $\theta$ por meio da equação (3). Com os valores de $\theta$ e utilizando a equação (4), encontramos os valores de $\lambda$ para $(m=1)$. A seguir apresentamos os resultados e discussões do experimento de Young em fenda dupla e da difração por um orifício circular.

\section{Resultados}

Foram realizadas quatro medidas por meio do experimento de Young. Cada medida foi realizada por um estudante diferente.

As tabelas 1 e 2 mostram os resultados obtidos na realização do experimento de Young para $m=1$ e $m=5$.

Tabela 1: Medidas da primeira faixa clara de ordem $1(\mathrm{~m}=1)$.

\begin{tabular}{|l|l|l|l|l|l|}
\hline & $\mathbf{D}(\mathbf{m})$ & $\mathbf{d}(\mathbf{m})$ & $\mathbf{y}(\mathbf{m})$ & $\boldsymbol{\theta}$ & $\boldsymbol{\lambda}(\mathbf{n m})$ \\
\hline $\mathbf{1}^{\mathbf{a}}$ medida & 5,575 & 0,002 & 0,0018 & 0,0185 & 645,74 \\
\hline $\mathbf{2}^{\mathbf{a}}$ medida & 5,575 & 0,002 & 0,0019 & 0,0205 & 681,61 \\
\hline $\mathbf{3}^{\mathbf{a}}$ medida & 5,576 & 0,002 & 0,0019 & 0,0195 & 681,49 \\
\hline $\mathbf{4}^{\mathbf{a}}$ medida & 5,574 & 0,002 & 0,0017 & 0,0175 & 609,97 \\
\hline Valor médio & $\mathbf{5 , 5 7 5}$ & $\mathbf{0 , 0 0 2}$ & $\mathbf{0 , 0 0 1 8}$ & $\mathbf{0 , 0 1 9 0}$ & $\mathbf{6 5 4 , 7 0}$ \\
\hline
\end{tabular}

Fonte: arquivo pessoal, 2014.

Tabela 2: Medidas da quinta faixa clara de ordem $5(m=5)$.

\begin{tabular}{|l|l|l|l|l|l|}
\hline & $\mathbf{D}(\mathbf{m})$ & $\mathbf{d}(\mathbf{m})$ & $\mathbf{y}(\mathbf{m})$ & $\boldsymbol{\theta}$ & $\boldsymbol{\lambda}(\mathbf{n m})$ \\
\hline $\mathbf{1}^{\mathbf{a}}$ medida & 5,575 & 0,002 & 0,0090 & 0,0925 & 645,74 \\
\hline $\mathbf{2}^{\mathbf{a}}$ medida & 5,575 & 0,002 & 0,0091 & 0,0935 & 652,91 \\
\hline $\mathbf{3}^{\mathbf{a}}$ medida & 5,576 & 0,002 & 0,0089 & 0,0915 & 638,85 \\
\hline $\mathbf{4}^{\mathbf{a}}$ medida & 5,574 & 0,002 & 0,0090 & 0,0925 & 645,85 \\
\hline Valor médio & $\mathbf{5 , 5 7 5}$ & $\mathbf{0 , 0 0 2}$ & $\mathbf{0 , 0 0 9 0}$ & $\mathbf{0 , 0 9 2 5}$ & $\mathbf{6 4 5 , 8 4}$ \\
\hline
\end{tabular}

Fonte: arquivo pessoal, 2014.

O valor do comprimento de onda informado no rótulo do laser é de $650 \mathrm{~nm}$ (n $\left.=10^{-9}\right)$. O valor médio do comprimento de onda considerando a tabela 1 e portanto $\mathrm{m}$ 
= 1 foi de 654,6 nm. Um desvio de apenas 4,6 nm em relação ao valor de referência, que corresponde à aproximadamente $0,71 \%$. Os resultados obtidos a partir da tabela 2 e considerando $m=5$ forneceu um valor médio de $645,84 \mathrm{~nm}$. Um desvio de apenas $4,2 \mathrm{~nm}$, que equivale a $0,65 \%$.

Para o experimento da difração por uma fenda circular, realizamos também quatro medidas, cada medida realizada por um estudante diferente.

Na tabela 3 mostramos os resultados obtidos no experimento da difração $m=1$.

Tabela 3: Medidas para o primeiro mínimo, primeiro anel escuro, de ordem $1(\mathrm{~m}=1)$.

\begin{tabular}{|l|l|l|l|l|l|}
\hline & $\mathbf{D}(\mathbf{m})$ & $\mathbf{d}(\mathbf{m})$ & $\mathbf{y}(\mathbf{m})$ & $\boldsymbol{\theta}$ & $\boldsymbol{\lambda}(\mathbf{n m})$ \\
\hline $\mathbf{1}^{\mathbf{a}}$ medida & 5,575 & 0,002 & 0,0022 & 0,0226 & 646,92 \\
\hline $\mathbf{2}^{\mathbf{a}}$ medida & 5,575 & 0,002 & 0,0024 & 0,0247 & 705,72 \\
\hline $\mathbf{3}^{\mathbf{a}}$ medida & 5,576 & 0,002 & 0,0021 & 0,0216 & 617,40 \\
\hline $\begin{array}{l}\mathbf{4}^{\mathbf{a}} \text { medida } \\
\text { Valor } \\
\text { médio }\end{array}$ & 5,574 & 0,002 & 0,0020 & 0,0206 & 588,21 \\
\hline
\end{tabular}

Fonte: arquivo pessoal, 2014.

A partir da tabela 3 , verificamos um valor médio de $639,56 \mathrm{~nm}$ para o comprimento de onda do laser. Comparando com o valor de referência de $650 \mathrm{~nm}$, o desvio foi de 10,44 nm, correspondendo, portanto a apenas 1,6\% do valor de referência.

Como justificativa pela escolha do relatório do grupo 1, abaixo apresentamos e interpretamos as figuras de difração, obtidas pelo os outros grupos de estudantes:

Figura 10: Experimento de Young

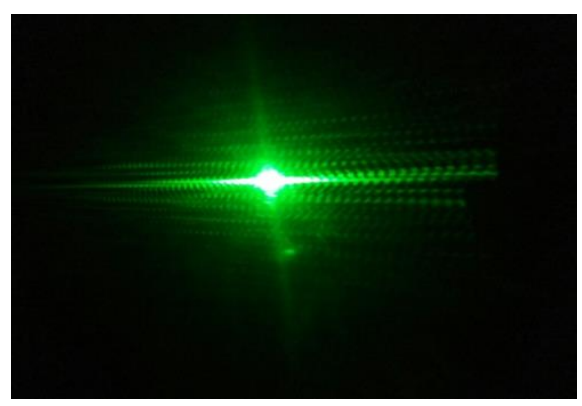

Fonte: arquivo pessoal, 2014. 
Figura 11: Fenda circular- grupo 2

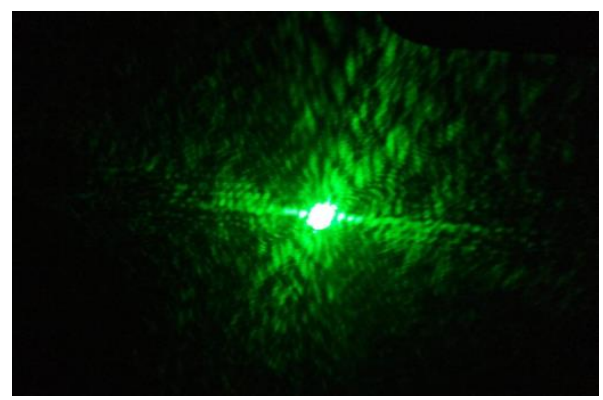

Fonte: arquivo pessoal, 2014.

Figura 12: Fenda circular- grupo 3

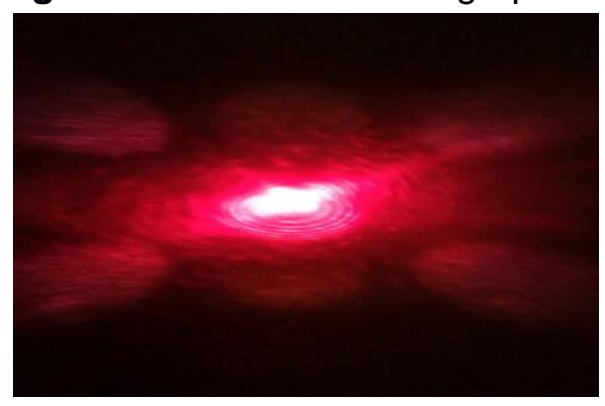

Fonte: arquivo pessoal, 2014.

Figura 13: Experimento de Young- grupo 4

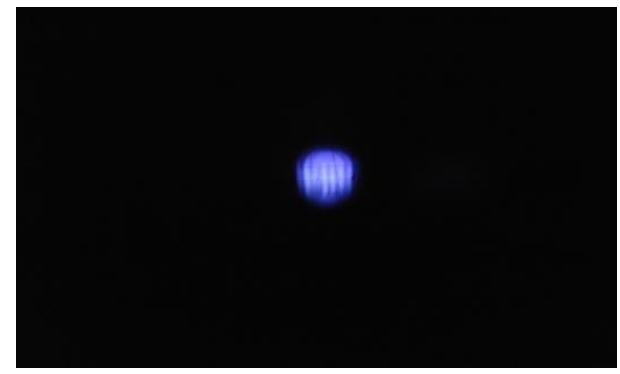

Fonte: arquivo pessoal, 2014.

Analisando a figura 10, obtida pelo grupo 2, nota-se que a imagem ficou trêmula e, portanto não foi possível identificar claramente as franjas claras e escuras. Isto aconteceu devido porque algumas vezes o laser se movimentava em torno do suporte, provocando assim, uma distorção na imagem observada na figura 10.

Na figura 11, que também foi obtida pelo grupo 2, foi feito um furo em uma folha de papel ofício. A luz emitida pelo laser, além de passar pelo furo atravessa também o papel, justificando o espalhamento da imagem. Os valores medidos do comprimento de onda foram: $\lambda=738 \mathrm{~nm}(\mathrm{~m}=5)$ e $624 \mathrm{~nm}$ para a difração pela fenda dupla e circular respectivamente. A medida do rótulo do laser era de $532 \mathrm{~nm}$, um erro de $38 \%$ e 17\% respectivamente. Na figura 14 é apresentado o material utilizado pelo grupo 2: 
Figura 14: Material utilizado pelo grupo 2

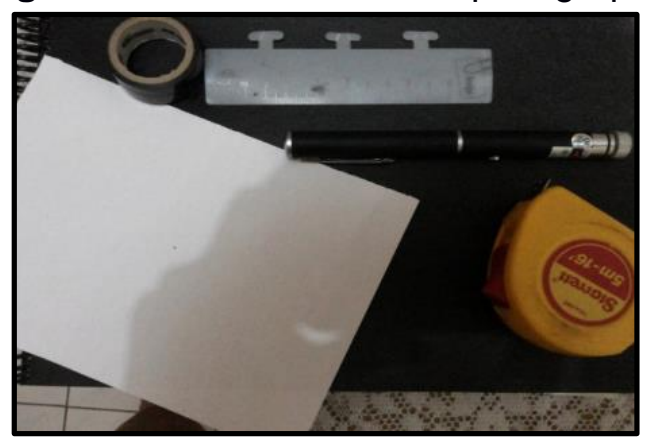

Fonte: arquivo pessoal, 2014.

Na figura 12, obtida pelo grupo 3, os estudantes fizeram um furo em uma folha de papelão. A utilização da folha de papelão sobrepôs regiões de máximo e mínimo da figura de interferência, prejudicando a visualização nítida das interferências construtiva e destrutiva. Entretanto, neste caso, o valor da medida apresentou boa concordância com o rótulo do laser: $647 \mathrm{~nm}$ (medida) e $650 \mathrm{~nm}$ (rótulo). Na figura 15 é apresentado o material utilizado pelo grupo 3:

Figura 15: Material utilizado pelo grupo3.

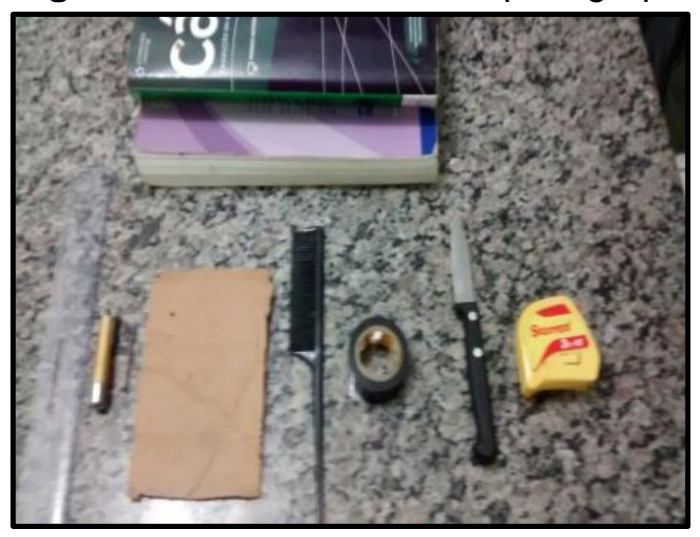

Fonte: arquivo pessoal, 2014.

Na figura 13 percebe-se a falta de nitidez das franjas claras e escuras. Isto ocorreu devido à espessura da fenda utilizada $(\mathrm{d}=5,7 \mathrm{~cm})$, a medida do ângulo $\theta=$ $5,3^{0}$ e a utilização de uma lanterna como fonte. Neste caso, o valor da medida do comprimento de onda foi de $\lambda=5,24 \mathrm{~mm}$; um erro da ordem de grandeza de $10^{4}$. $\mathrm{Na}$ figura 16 é apresentado o material utilizado pelo grupo 4: 
Figura 16: Material utilizado pelo grupo 4

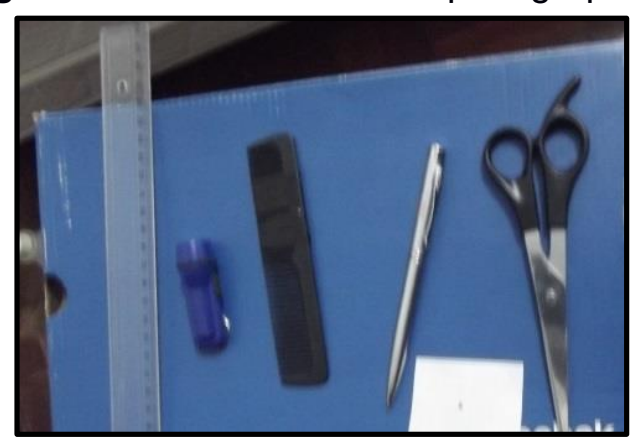

Fonte: arquivo pessoal, 2014.

\section{Considerações finais}

Para $\mathrm{m}=1$, o experimento de Young forneceu o valor médio de 654,70 nm para o comprimento de onda, um desvio de apenas $0,71 \%$ em relação ao valor de referência de $650 \mathrm{~nm}$. Para $\mathrm{m}=5$, o valor obtido foi de 645,84 nm, com um desvio de apenas $0,65 \%$.

O experimento de difração por uma fenda circular forneceu um valor médio de $639,56 \mathrm{~nm}$ para o comprimento de onda, um desvio de apenas 1,6\% em relação ao valor de referência.

Concluímos que, mesmo realizando experimentos com materiais de baixo custo, é possível obter uma informação com uma precisão razoável no nível de escala da nanociência (fenômenos que ocorrem da ordem de $10^{-9} \mathrm{~m}$ ).

O experimento de Young e o de difração reproduzidas na sala de aula promoveram uma participação ativa dos estudantes durante a construção da atividade prática e ajudou na compreensão da teoria relacionada ao comportamento da luz:

\footnotetext{
"Através da pratica de Young, conseguimos interagir com as nossas próprias dúvidas sobre a dualidade onda-partícula da luz, chegando a conclusões mais reais que os meros números e formulas" (estudante1).

"Foi de extrema importância a realização desse experimento, pois tornou concreto o que antes era abstrato, colaborando com a construção do nosso conhecimento" (estudante 2)

"Sendo uma disciplina bastante abstrata, a física moderna, é difícil de compreender apenas na teoria, porem se unir a pratica e a teoria, tudo fica mais fácil, como nesse experimento, que sendo simples de efetuálo foi possível um entendimento mais concreto e eficaz" (estudante 3 ).
} 
Esperamos que a leitura desse relato de prática incentive professores e estudantes a construírem experimentos de física para aprimorar os conceitos da óptica geométrica e o princípio de superposição de ondas. Pode-se ampliar o horizonte dos conhecimentos dos estudantes para o entendimento não somente das posições de alguns máximos e mínimos do padrão de interferência, mas conduzi-los a compreender e determinar as intensidades relativas dos máximos e mínimos em relação ao máximo central (vide: Grober et. al., 2014). Igualmente, este trabalho poderá ser expandido para a construção de atividades práticas que envolvam a formação de figuras de interferências originadas por meio de gotas esféricas de água ou tubos capilares (vide: Hou, Zhao, Xiao, 2012).

As dificuldades encontradas pelos grupos 2, 3 e 4 para a obtenção das figuras nítidas de interferência pode alertar o professor a evitar alguns procedimentos e materiais quando for replicar o experimento de difração com seus estudantes. Recomenda-se afixar bem o laser no suporte e não fazer o orifício em papelão ou folha de papel de ofício branca, na prática da fenda circular.

Por fim, em um trabalho ulterior, pretendemos discutir episódios históricos que elucidam as controvérsias entre a teoria corpuscular da luz e a ondulatória, levando o estudante a um entendimento de alguns aspectos da natureza da ciência como o caráter mutável e dinâmico da ciência, as influências sociais, políticas e econômicas que circunscrevem a ciência e a falibilidade dos cientistas.

\section{REFERÊNCIAS}

BARBOSA, V.C; BREITSCHAFT, A. M. S; MENDONÇA, J. P.R, MOREIRA, L. M; MORAES, P. C. G. Uma visualização do principio de Huygens-Fresnel na obtenção de um padrão de difração. Revista Brasileira de Ensino de Física, v. 34, n. 3, 2012.

BORN, M.; WOLF, E. Principles of Optics - Electromagnetic Theory of Propagation, Interference and Diffraction of Light. $7^{\text {a }}$ ed. expandida. Cambridge University Press, Cambridge, 1999. 936p.

GROBER, S.; VETTER, M.ECKERT, B.; JODL, H. J. Diffraction and interference - a standard teaching topic using non-standard diffracting objects. European Journal of Physics, v. 35, n. 015003, p.1-12, 2014.

HALLIDAY, D. RESNICK, R. WALKER, J. Fundamentos de Física: Óptica e Física Moderna. $8^{a}$ ed. Tradução e revisão técnica: Ronaldo Sérgio de Biasi. Rio de Janeiro: LTC, 2009. 438p. 
HOU, Z.; ZHAO; X.; XIAO, J. A simple double-source model for interference of capillaries. European Journal of Physics, v. 33, p.199-206, 2012.

MARINHO, R.F; VAILANT, C. C. R; COSTACO, I. L.L; SILVA, L. P. V. Medidas dos comprimentos de onda do espectro visível: uma proposta didática de baixo custo. (2015) Disponível

em: http://www.ifg.edu.br/jatai/semlic/seer/index.php/anais/article/viewArticle/407. Acesso em 27 ago. 2016.

RIBEIRO; J. L. P.; VERDEAUX, M. F. S. Atividades experimentais no ensino de óptica: uma revisão. Revista Brasileira de Ensino de Física, v. 34, n. 4, 2012.

SILVA, B. V. C. Young fez, realmente, o experimento da fenda dupla? Lat. Am. J. Phys. Educ, v.3, n. 2, 2009.

SILVA, L.F; ASSIS, A. A física moderna no ensino médio: um experimento para abordar o efeito fotoelétrico. Caderno Brasileiro de Ensino de Física, v. 29, n. 2: p. 313-324, ago. 2012.

SOUZA, L.A; DA SILVA, L; HUGUENIN, J. A.O; BALTHAZAR, W. F. Discutindo a natureza ondulatória da luz e o modelo da óptica geométrica através de uma atividade experimental de baixo custo. Revista Brasileira de Ensino de Física, v. 37, n. 4, 2015. 